\title{
Spin-Polarized Transport through Quantum Dots in the Cotunneling Regime
}

\author{
I. WEYMANN
}

Department of Physics, Adam Mickiewicz University

Umultowska 85, 61-614 Poznań, Poland

\begin{abstract}
The spin-polarized transport through two-level quantum dots weakly coupled to ferromagnetic leads is considered theoretically in the Coulomb blockade regime. It is assumed that the dot is doubly occupied, so that the current flows due to cotunneling through singlet and triplet states of the dot. It is shown that, by sweeping the bias voltage, one can induce a singlet-triplet transition, which depends on the magnetic configuration of the system and leads to nontrivial bias dependence of both the differential conductance and tunnel magnetoresistance.
\end{abstract}

PACS numbers: 72.25.Mk, 73.63.Kv, 85.75.-d, 73.23.Hk

\section{Introduction}

Transport properties of quantum dots coupled to ferromagnetic leads have been a subject of thorough studies since a few years [1-3]. This is because such systems are considered to play an important role in future spintronics devices, for storing and processing information. Furthermore, they enable the study and manipulation of single spins and charges. An interesting aspect of spin-polarized transport through quantum dots is the problem of cotunneling through quantum dots hosting two orbital levels. In the Coulomb blockade regime and when the dot is occupied by two electrons, the current flows then due to second-order tunneling processes (cotunneling), taking place through singlet and triplet states of the quantum dot. We show that by changing the bias voltage one can induce a singlet-triplet transition in such systems. This transition is more pronounced in the antiparallel configuration, and leads to interesting bias dependence of both the differential conductance $G$ and tunnel magnetoresistance (TMR).

\section{Model and method}

The system considered in this paper consists of two-level quantum dot coupled to ferromagnetic leads with spin polarization $p$. The magnetizations of 
the leads can form either parallel or antiparallel magnetic configuration. Due to the coupling to the leads, the dot levels acquire certain width, described by the parameter $\Gamma[3]$. The Hamiltonian $H$ of the system involves four terms, $H=H_{\mathrm{L}}+H_{\mathrm{R}}+H_{\mathrm{D}}+H_{\mathrm{T}}$, where the first two terms describe noninteracting electrons in the leads and the last term accounts for tunneling processes between the dot and leads [3]. The quantum dot is described by $H_{\mathrm{D}}$, which reads [4]:

$$
\begin{aligned}
H_{\mathrm{D}} & =\sum_{j \sigma} \varepsilon_{j} n_{j \sigma}+U \sum_{j} n_{j \uparrow} n_{j \downarrow}+U^{\prime} \sum_{\sigma \sigma^{\prime}} n_{1 \sigma} n_{2 \sigma^{\prime}} \\
& -J \sum_{\alpha \beta \gamma \delta} d_{1 \alpha}^{\dagger} d_{1 \beta} d_{2 \gamma}^{\dagger} d_{2 \delta} \boldsymbol{\sigma}_{\alpha \beta} \boldsymbol{\sigma}_{\gamma \delta},
\end{aligned}
$$

where $n_{j \sigma}=d_{j \sigma}^{\dagger} d_{j \sigma}$ and $d_{j \sigma}^{\dagger}\left(d_{j \sigma}\right)$ is the creation (annihilation) operator of an electron with spin $\sigma$ on the $j$-th level $(j=1,2)$, while $\varepsilon_{j}$ is the corresponding energy. On-level and inter-level Coulomb interaction between electrons is described by $U$ and $U^{\prime}$, respectively. The last term in $H_{\mathrm{D}}$ corresponds to the exchange energy due to the Hund rule, with $J$ being the respective exchange coupling and $\boldsymbol{\sigma}$ denoting a vector of the Pauli spin matrices.

In order to find the current flowing through the dot in the Coulomb blockade, one has to calculate the respective cotunneling rates. Within the second-order perturbation theory, the rate of a cotunneling process from lead $r$ to lead $r^{\prime}$ which changes the dot state from $|\chi\rangle$ into $\left|\chi^{\prime}\right\rangle$ is given by

$$
\gamma_{\mathrm{rr}^{\prime}}^{\chi \rightarrow \chi^{\prime}}=\frac{2 \pi}{\hbar}\left|\sum_{v} \frac{\left\langle\Phi_{r^{\prime}}^{\chi^{\prime}}\left|H_{\mathrm{T}}\right| \Phi_{v}\right\rangle\left\langle\Phi_{v}\left|H_{\mathrm{T}}\right| \Phi_{r}^{\chi}\right\rangle}{\varepsilon_{\mathrm{i}}-\varepsilon_{v}}\right|^{2} \delta\left(\varepsilon_{\mathrm{i}}-\varepsilon_{\mathrm{f}}\right),
$$

with $\varepsilon_{\mathrm{i}}$ and $\varepsilon_{\mathrm{f}}$ denoting the energies of initial and final states, $\left|\Phi_{r}^{\chi}\right\rangle$ being the state of the system with an electron in the lead $r$ and the dot in state $|\chi\rangle$, whereas $\left|\Phi_{v}\right\rangle$ is a virtual state with energy $\varepsilon_{v}$. The cotunneling current flowing through the system from the left to right lead is then given by $I=e \sum_{\chi \chi^{\prime}} P_{\chi}\left(\gamma_{\mathrm{LR}}^{\chi \rightarrow \chi^{\prime}}-\gamma_{\mathrm{RL}}^{\chi \rightarrow \chi^{\prime}}\right)$, where $P_{\chi}$ denotes the corresponding occupation probability. The occupation probabilities can be found from the following master equation: $0=\sum_{r r^{\prime}} \sum_{\chi^{\prime}}\left(-\gamma_{r r^{\prime}}^{\chi \rightarrow \chi^{\prime}} P_{\chi}+\gamma_{r r^{\prime}}^{\chi^{\prime} \rightarrow \chi} P_{\chi^{\prime}}\right)$, together with the normalization condition $\sum_{\chi} P_{\chi}=1$.

In particular, we consider the case when the dot is doubly occupied at equilibrium and the system is in the Coulomb blockade regime. For assumed parameters, there are four different two-particle states in the dot possible, these are: a singlet $|S=0, M=0\rangle=|\uparrow \downarrow\rangle|0\rangle$, and three triplets $|1,0\rangle=(|\uparrow\rangle|\downarrow\rangle+|\downarrow\rangle|\uparrow\rangle) / \sqrt{2}$, $|1,1\rangle=(|\uparrow\rangle|\uparrow\rangle$ and $|1,-1\rangle=|\downarrow\rangle|\downarrow\rangle$, where the first (second) ket corresponds to the first (second) orbital level of the dot. 


\section{Results and discussion}

Figure 1 presents the bias voltage dependence of differential conductance $G$ and TMR. The TMR is defined as $\mathrm{TMR}=I_{\mathrm{P}} / I_{\mathrm{AP}}-1$, where $I_{\mathrm{P}}\left(I_{\mathrm{AP}}\right)$ is the current flowing through the system in the parallel (antiparallel) magnetic configuration. For the assumed parameters, the energy difference between the singlet and triplet states, $\Delta_{\mathrm{ST}}=J+\varepsilon_{1}-\varepsilon_{2}$, is $\Delta_{\mathrm{ST}}<0$. As a consequence, the dot is occupied by two electrons on the lowest energy level, $|0,0\rangle=|\uparrow \downarrow| 0\rangle$, and the ground state is a singlet, $S=0$. For low bias voltages, the current flows thus due to elastic cotunneling processes through the $S=0$ state. However, once $|e V| \gtrsim\left|\Delta_{\mathrm{ST}}\right|$, the triplet states start participating in transport, leading to an increase in the differential conductance at $|e V| \approx\left|\Delta_{\mathrm{ST}}\right|$. In other words, the suppression of cotunneling through $S=1$ states gives rise to a minimum in differential conductance which is present in both magnetic configurations of the system, see Fig. 1a. In addition, one
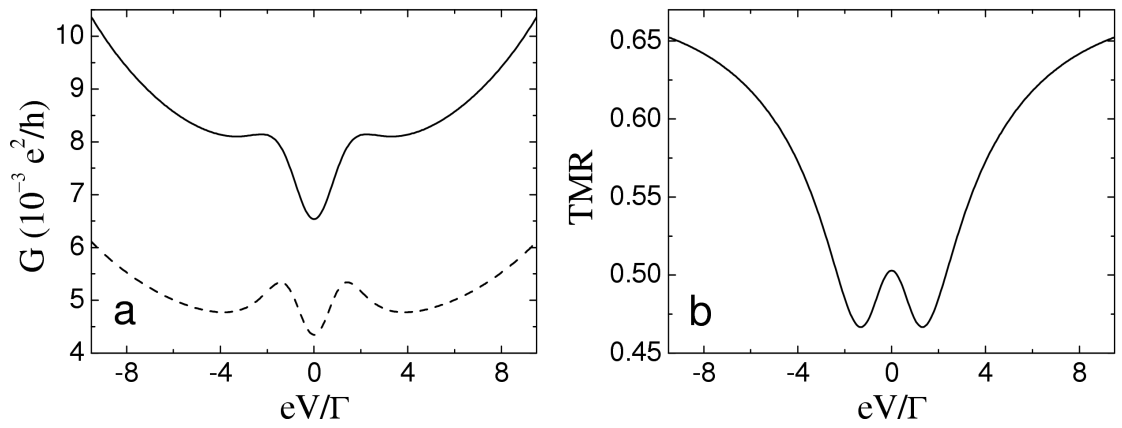

Fig. 1. The bias dependence of the differential conductance (a) in the parallel (solid line) and antiparallel (dashed line) configuration and the TMR (b) for parameters: $k_{\mathrm{B}} T=0.4 \Gamma, \varepsilon_{1}=-50 \Gamma, \varepsilon_{2}=-46 \Gamma, U=U^{\prime}=30 \Gamma, J=3 \Gamma$ and $p=0.5$.

can see that $G$ in the antiparallel configuration displays two small peaks appearing at $|e V| \approx\left|\Delta_{\mathrm{ST}}\right|$. Such behavior results from the fact that at this bias voltage the triplet states start participating in transport and, due to the spin asymmetry in tunneling processes, nonequilibrium spin accumulation, $P_{|1,1\rangle} \neq P_{|1,-1\rangle}$, is built up in the dot. The spin accumulation increases with the bias voltage which leads to a decrease in cotunneling through the most favorable states [3]. This leads to suppression of $G$ in the antiparallel configuration and to two local minima, see Fig. 1a.

Another feature visible in Fig. 1a is the difference in the conductance for the parallel and antiparallel magnetic configurations, which results from the spin asymmetry of cotunneling processes. This difference leads to non-zero TMR effect shown in Fig. 1b. The TMR exhibits a local maximum in the zero bias and two satellites local minima for $|\mathrm{eV}| \approx\left|\Delta_{\mathrm{ST}}\right|$. On the other hand, for $|\mathrm{eV}|>\left|\Delta_{\mathrm{ST}}\right|$, TMR approaches the Julliere value [5]. 

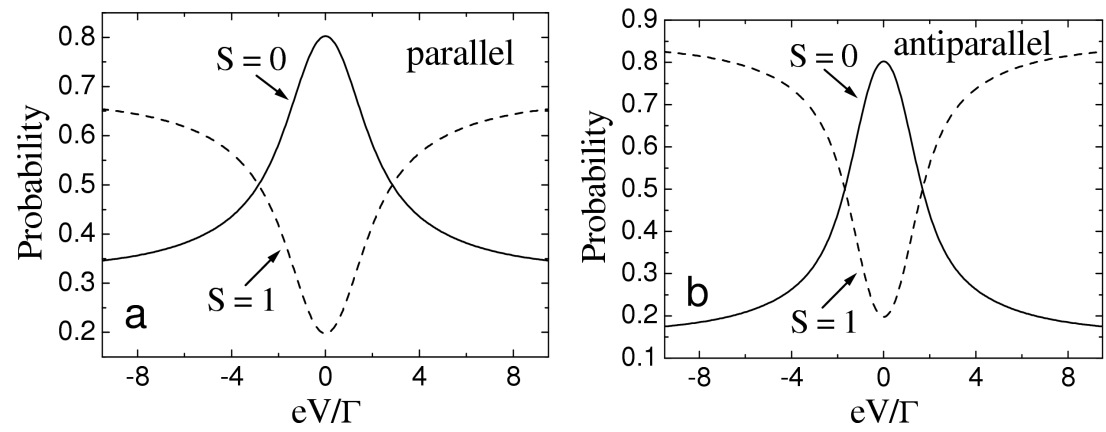

Fig. 2. The bias dependence of the probability of occupation of singlet and triplet quantum dot states in the parallel (a) and antiparallel (b) magnetic configuration for parameters the same as in Fig. 1. A singlet-triplet transition is clearly visible.

In Fig. 2 we also show the probability of occupation of singlet and triplet dot states. For low bias voltages the dot is in the $S=0$ state, irrespective of magnetic configuration. When increasing the bias voltage, the state of the dot changes into a triplet, $S=1$. This singlet-triplet transition is more pronounced in the antiparallel configuration, see Fig. 2b. Furthermore, in the antiparallel configuration it takes place for lower bias voltages than in the parallel one. This bias voltage-induced singlet-triplet transition may be of importance for future spintronics and magnetoelectronics devices.

\section{Acknowledgments}

This work, as part of the European Science Foundation EUROCORES Programme SPINTRA, was supported by funds from Ministry of Science and Higher Education as a research project in years 2006-2009 and the EC Sixth Framework Programme, under contract No. ERAS-CT-2003-980409, and the Foundation for Polish Science.

\section{References}

[1] S. Maekawa, T. Shinjo, Spin Dependent Transport in Magnetic Nanostructures, Taylor Francis, London 2002.

[2] W. Rudziński, J. Barnaś, Phys. Rev. B 64, 085318 (2001); M. Braun, J. König, J. Martinek, Phys. Rev. B 70, 195345 (2004).

[3] I. Weymann, J. König, J. Martinek, J. Barnaś, G. Schön, Phys. Rev. B 72, 115334 (2005); I. Weymann, Europhys. Lett. 76, 1200 (2006); I. Weymann, J. Barnaś, Phys. Rev. B 75, 155308 (2007).

[4] W. Izumida, O. Sakai, S. Tarucha, Phys. Rev. Lett. 87, 216803 (2001).

[5] M. Julliere, Phys. Lett. A 54, 225 (1975). 